\title{
Correlation of Hemostatic Parameters with Poly (ADP-ribose) Polymerase-1 (PARP-1) Polymorphisms, Mutations, Laboratory, and Clinical Characteristics in 114 Patients with Philadelphia-Negative Myeloproliferative Neoplasms
}

Giannakopoulou $\mathrm{N}^{* 1}$, Diamantopoulos $\mathrm{P}^{1}$, Politou $\mathrm{M}^{2}$, Kontandreopoulou C-N ${ }^{1}$, Zoi $\mathrm{K}^{3}$, Giannopoulos $\mathrm{A}^{3}$, Korakakis $\mathrm{D}^{2}$, Kassi $T^{2}$, Chatzidavid $S^{1}$, Mantzourani $M^{1}$, Dimou $M^{4}$, Kyriakakis $G^{1}$ and Viniou $N-A^{1}$

${ }^{1}$ Hematology Unit, First Department of Internal Medicine, Laiko General Hospital, School of Medicine, National and Kapodistrian University of Athens, Athens, Greece

${ }^{2}$ Hematology Laboratory Blood Bank, Aretaieion Hospital, School of Medicine, National and Kapodistrian University of Athens, Athens, Greece

${ }^{3}$ Hematology Research Laboratory, Biomedical Research Foundation, Academy of Athens, Athens, Greece

${ }^{4}$ First Propedeutic Department of Internal Medicine, Laiko General Hospital, School of Medicine, National and Kapodistrian University of Athens, Athens, Greece

*Corresponding author: Giannakopoulou N, Hematology Unit, First Department of Internal Medicine, Laiko General Hospital, School of Medicine, National and Kapodistrian University of Athens, 11527 Athens, Greece, Tel: +30 6974382235; +30 6976776260, E-mail: nef_gia@yahoo.gr

Citation: Giannakopoulou N, Diamantopoulos P, Politou M, Kontandreopoulou C-N, Zoi K, et al. (2021) Correlation of Hemostatic Parameters with Poly (ADP-ribose) Polymerase-1 (PARP-1) Polymorphisms, Mutations, Laboratory, and Clinical Characteristics in 114 Patients with Philadelphia-Negative Myeloproliferative Neoplasms. J Hematol Blood Disord 7(1): 103

Received Date: July 18, 2021, Accepted Date: August 11, 2021, Published Date: August 13, 2021

\begin{abstract}
Patients with Philadelphia-negative myeloproliferative neoplasms (PN-MPN) are at a higher risk for venous thrombosis. Thromboelastometry may prove efficient to evaluate the patient's thrombotic risk. In this study, based on data from 114 patients with PN-MPN from a single center in Greece, hemostatic profile was assessed with routine coagulation tests, Rotational Thromboelastometry (ROTEM ${ }^{\circ}$ ), and Platelet Function Analyzer (PFA)-100 and correlated with clinical, laboratory, treatment characteristics, gene mutations and polymorphisms of poly (ADP-ribose) polymerase-1 (PARP-1). According to thromboelastometry parameters, patients with essential thrombocythemia (ET) had a more hypercoagulable status compared to patients with polycythemia vera (PV) and myelofibrosis (MF) while in all patients there was a statistically positive correlation between alpha angle and platelet (PLT) count and between maximum clot firmness (MCF) and PLT count $(\mathrm{p}<0.001)$. In Janus kinase $2(J A K 2)$ positive patients with PV, the absence of the PARP-1 polymorphism was correlated with thrombosis during follow-up ( $\mathrm{p}=0.019)$. Among 47 patients under treatment with aspirin and complete platelet inhibition, no patient had a thrombotic episode in follow-up period $(\mathrm{p}=0.006)$. Based on our results, global hemostatic assays in combination with other parameters could help identify patients with PN-MPN at higher thrombotic risk and adjust the type and dose of appropriate treatment.
\end{abstract}

Keywords: Philadelphia-Negative Myeloproliferative Neoplasms; Thrombosis; Thromboelastometry; Poly (ADP-ribose) Polymerase-1 


\section{Introduction}

Myeloproliferative neoplasms (MPN) are clonal disorders of hematopoietic stem cells characterized by proliferation of one or more myeloid cell lines (granulocytic, erythroid, megakaryocytic). According to the revised 2016 World Health Organization (WHO) classification, PN-MPN include three main disease subgroups: PV, ET, and MF [1]. PN-MPNs can be driven by several genetic mutations, including mutations of the JAK2 gene, the trombopoietin gene $(M P L)$, and the calreticulin gene (CALR). In addition, further mutations in other genes, such as epigenetic modification genes, have been found in PN-MPN. These genes include mainly RNA splicing genes (SF3B1, SRSF2, U2AF1), chromatin histone modification genes (ASXL1, EZH2), and DNA methylation genes (DNMT3A, IDH1, IDH2, TET2) [2,3]. Patients with PN-MPN are at increased risk for arterial and venous thrombosis [4]. Due to the morbidity and mortality of these events, antiplatelet and/or anticoagulant agents are commonly employed as primary and/or secondary prophylaxis [5].

Patients with PN-MPN commonly present with abnormalities in laboratory coagulation tests that are consistent with a hypercoagulable state [6]. Thromboelastometry is a global coagulation assay performed in whole blood that can evaluate the contribution of clotting factors, platelets, and fibrinogen in the clotting process and may prove efficient to evaluate the patient's thrombotic risk [7]. To date, few studies have evaluated the thromboelastometry profile of MPN patients [8-11].

PARP-1 is a nuclear chromatin associated enzyme involved in several important cellular processes, particularly in DNA repair. Even though rs1136410:C>T or V762A polymorphism of PARP-1 is the most studied single nucleotide polymorphism (SNP) in the PARP-1 gene and probably involved in human carcinogenesis, results from previous studies concerning associations between PARP-1 polymorphisms and MPN risk are inconclusive [12]. We decided to examine PARP-1 in our study because it constitutes a molecule of great interest for our group, both for its role as a prognostic marker in myelodysplastic syndromes and as a possible therapeutic target as described in publications of our team [13,14], [Kontandreopoulou, et al., in press in Blood Advances]. Taking into consideration a numerous of studies which have tried to elucidate the potential involvement of the PARP-1 rs1136410 polymorphism in several cancers (gastric, cervical, lung and breast cancers) [12,15,16], our aim was to evaluate the possible role of this base excision repair gene variant in PN-MPN. Moreover, the possible association between this polymorphism and thrombotic risk could stand, taking into account the protective role that was found in coronary artery disease in a Chinese population [17].

The aim of the present study was to examine the hemostatic profile of patients with PN-MPN and correlate it with clinical, laboratory, and treatment characteristics, JAK2, MPL, CALR gene mutations, and the polymorphism rs1136410:C>T (V762A) of PARP-1.

\section{Materials and Methods}

The present study included patients from one University Hospital in Greece, with a confirmed diagnosis of PN-MPN per the 2016 revision to the World Health Organization classification of myeloid neoplasms and acute leukemia. A written informed consent was obtained by all patients and the demographic, epidemiologic, clinical, and laboratory characteristics of the patients were recorded. History of thromboembolic events and occurrence during follow-up were also recorded along with the treatment regimens administered for the management of PN-MPN, including antiplatelet and anticoagulation treatment. At the time of enrollment, all patients were assessed with a complete blood count, routine coagulation tests, such as Prothrombin Time (PT), International Normalized Ratio (INR), activated Partial Thromboplastin Time (aPTT), fibrinogen, and D-Dimers, analyzed with the automatic coagulation analyzer Sysmex (Siemens, Berlin, Germany)]. Furthermore, at the time of sample collection all patients were examined with ROTEM (Munich, Germany), specifically EXTEM as a screening test for the extrinsic hemostasis system, and several parameters such as clotting time (CT), clot formation time (CFT), MCF, alpha angle, lysis index at 30 minutes (LI30) and lysis index at 60 minutes (LI60) were calculated as described in Figure 1 [18]. 


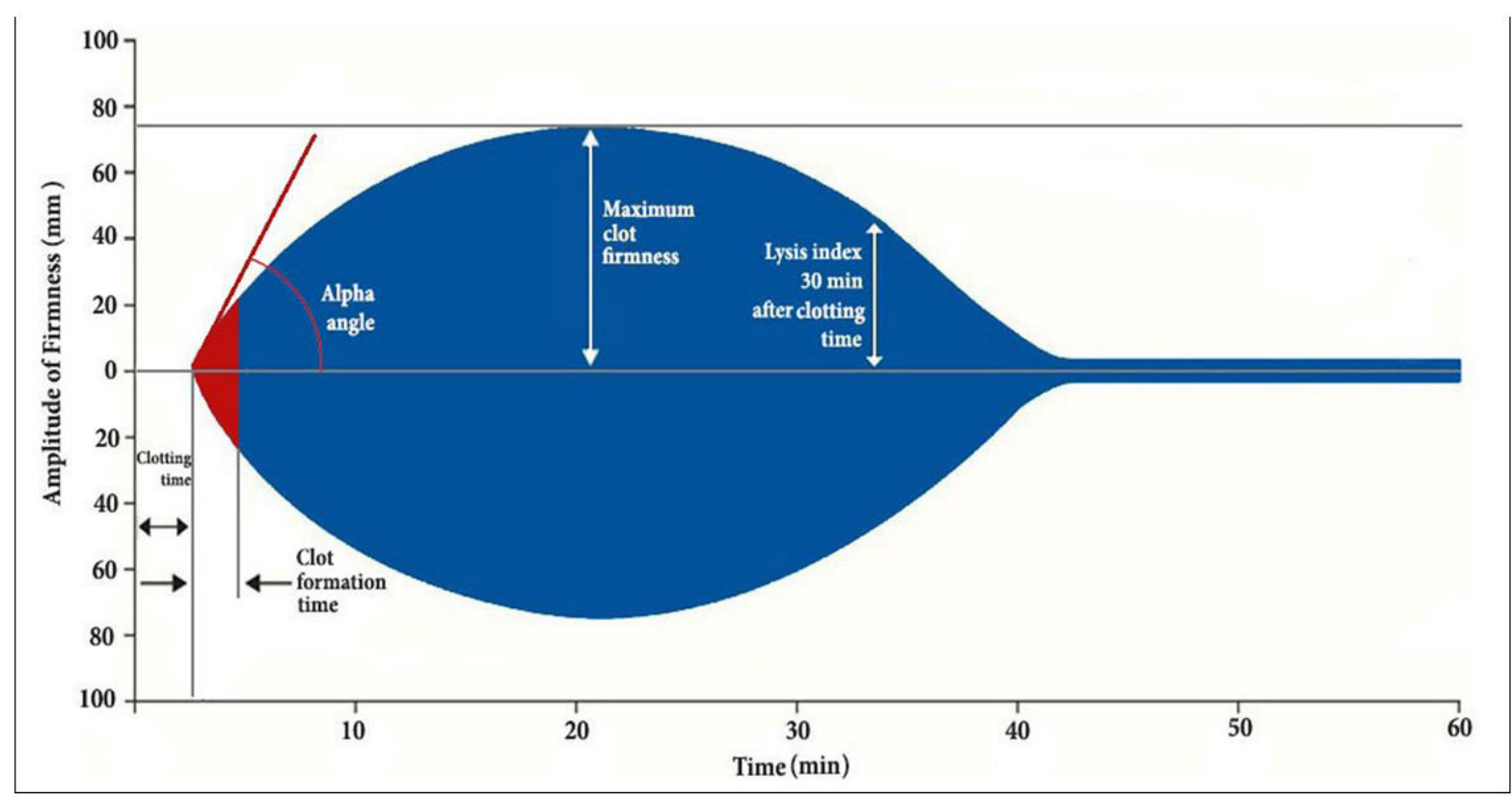

Figure 1: ROTEM parameters

Clotting Time (CT): corresponds to the time from the beginning of the analysis until the start of clot formation. Shortening of CT indicates hypercoagulability (reference values 38-79 sec); Clot Formation time (CFT): the time from the initiation of clotting until a clot firmness of $20 \mathrm{~mm}$ is detected. Shortening of CFT indicates hypercoagulability (34$159 \mathrm{sec}$ ); maximum clot firmness (MCF): represents clot firmness and overall clot stability. A low MCF represents a weak clot with severe bleeding risk (50-72 $\mathrm{mm}$ ); alpha angle: the angle of tangent between $0 \mathrm{~mm}$ and the curve when the clot firmness is $20 \mathrm{~mm}$. The more acute it is the more hypocoagulable the patient's state; Lysis Index at 30 and 60 minutes (LI30), (LI60): the percentage of remaining clot stability in relation to the MCF value at 30 and 60 minutes after CT respectively. A low LI value indicates hyperfibrinolysis (reference values > 15\%)

A platelet function assay was performed with PFA-100 using collagen-epinephrine (COL/EPI) and collagen-adenosine diphosphate (COL/ADP) cartridges. Closure time was defined as prolonged if $>150 \mathrm{sec}$ for COL/EPI or $>100 \mathrm{sec}$ for COL/ADP. A closure time $>300$ sec demonstrated optimal platelet inhibition with aspirin and clopidogrel and a value $>150 \mathrm{sec}$ a satisfactory platelet inhibition. Finally, all patients were tested for JAK2, MPL and CALR mutations and the PARP-1 rs1136410 polymorphism.

\section{Mutational analysis of PN-MPN tested genes}

Mutational analysis of JAK2, MPL and CALR genes was performed on peripheral blood DNA. JAK2 and MPL mutations were detected using standard PCR assays (sensitivity $>1 \%$ ) and CALR mutations were detected using a high-resolution melting analysis (HRMA)-PCR assay (sensitivity>2.5\%) [19-21]. The results were confirmed by Sanger sequencing analysis.

\section{Detection and genotyping of PARP-1 rs1136410 polymorphism}

For the detection of the PARP-1 polymorphism, samples were collected into ethylenediamine tetracetic acid (EDTA) tubes and processed within 4 hours from collection. Genomic DNA was extracted from cells of peripheral blood sample using a commercially available kit according to the manufacturer's protocol (Purelink Genomic DNA Mini Kit, Invitrogen, CA, USA). Genotyping of rs1136410 polymorphism consisted of a qualitative PCR followed by an allele-specific restriction enzyme digestion. The primers that were used (5'-GCCATTCACTGTGTTGGACCTT-3' and 5'-TTGACATCGATGGGATCCTT-3') amplified a 211 base pair (bp) fragment creating an Aci restriction site in the PARP-1-762A allele. The PCR was conducted as follows: $94{ }^{\circ} \mathrm{C}$ for 5 minutes, followed by 30 cycles of $94{ }^{\circ} \mathrm{C}$ for 1 minute, $55^{\circ} \mathrm{C}$ for 1 minute, $72{ }^{\circ} \mathrm{C}$ for 1 minute, and a final extension cycle at $72{ }^{\circ} \mathrm{C}$ for 10 
minutes. The amplification product was evaluated after electrophoresis in $2 \%$ agarose gel stained with ethidium bromide. The amplified DNA was then digested with the AciI restriction enzyme (New England Biolabs, Massachusetts, USA) at $37{ }^{\circ} \mathrm{C}$ for 30 minutes. The digestion products were evaluated by polyacrylamide gel electrophoresis in $8 \%$ gel (at $85 \mathrm{~V}$ for 2.5 hours). For homozygous PARP-1-762V patients, one undigested band (211bp) was visualized. In heterozygous patients, three bands (211 bp, $117 \mathrm{bp}, 94 \mathrm{bp}$ ) were visible, whereas for homozygous PARP-1-762A patients only two bands (117 bp and 94 bp) were seen.

\section{Statistical analysis}

Statistical analysis was performed using IBM SPSS statistics, version 23.0 (IBM Corporation, North Castle, NY, USA). The MannWhitney $\mathrm{U}$ test and the Kruskal-Wallis test were used to check for differences in continuous variables between two or more than two groups respectively. The Chi-squared test was used for correlations between categorical variables. A p-value under 0.05 was considered statistically significant. Two-sided p-values are reported.

\section{Results}

\section{Patients' characteristics}

From April 2016 to March 2020, we enrolled consecutively 114 patients (32 with PV, 70 with ET, and 12 with MF) with a median age of 63 years (15-86) as well as 68 healthy subjects to be used as controls only for the SNP/PARP-1 analysis. Their demographic and basic clinical, molecular, and treatment characteristics are shown in Table 1. All participants were followed up until December 2020, with a median follow-up time of 34 months (9-57 months).

\begin{tabular}{|l|l|}
\hline Characteristics & Result \\
\hline Number of patients, N (\%) & $114(100)$ \\
\hline Sex (Male), N (\%) & $58(50.8)$ \\
\hline Age (years), median (range) & $63(15-86)$ \\
\hline Diagnosis, N (\%) & $114(100)$ \\
\hline PV & $32(28)$ \\
\hline ET & $70(61.4)$ \\
\hline MF & $12(10.5)$ \\
\hline Antiplatelet therapy, N (\%) & $82(71.9)$ \\
\hline Anticoagulant therapy, N (\%) & $13(11.4)$ \\
\hline Thrombosis before diagnosis, N (\%) & $20(17.5)$ \\
\hline Thrombosis after diagnosis, N (\%) & $13(11.4)$ \\
\hline Mutation status, N (\%) & \\
\hline JAK2 & $59(51.8)$ \\
\hline CALR & $14(14)$ \\
\hline MPL & $4(3.8)$ \\
\hline Triple negative & $37(32.4)$ \\
\hline PARP-1 polymorphism, N (\%) & $28(26.7)$ \\
\hline Absent & $77(73.3)$ \\
\hline Heterozygous & $26(24.8)$ \\
\hline Homozygous & $2(1.9)$ \\
\hline
\end{tabular}

PV, polycythemia vera; ET, essential thrombocythemia; MF, myelofibrosis

Table 1: Patients' demographic, clinical, therapy, and molecular characteristics at the time of sample collection 
Regarding the mutational status, 59 (51.8\%) patients were JAK2 positive, 14 (14\%) CALR positive, 4 (3.8\%) MPL positive, and 37 $(32.4 \%)$ triple negative for all these mutations.

At the time of sample collection, 69 (60.5\%) patients were under treatment with hydroxyurea (HU), 21 (18.6\%) with anagrelide, 12 $(10,5 \%)$ with ruxolitinib, $3(2.6 \%)$ with interferon alpha, and $5(4.4 \%)$ with an alkylating agent. 82/114 patients were treated with antiplatelets [ aspirin 73 (64\%), clopidogrel 2 (1.8\%), a combination of the two 7 (6.1\%)] while 13 patients were under anticoagulant therapy [a vitamin K antagonist 2 (1.8\%), a Xa-inhibitor 6 (5.3\%), and low-molecular-weight heparin 5 (4.4\%)].

At baseline (i.e., before the diagnosis of PN-MPN), 20 (17,5\%) patients had a thrombotic episode [6, ischemic stroke; 5, coronary artery disease (CAD); 2, deep vein thrombosis (DVT); 1, carotid stenosis; 1, a combination of ischemic stroke and CAD; 2, pulmonary embolism (PE); 3, visceral thrombosis].

After diagnosis of PN-MPN, 13 (11.4\%) patients developed a thrombotic episode, all of them for the first time (3, ischemic stroke; 3. CAD; 4, DVT; 1, a combination of stroke and CAD; 1, a combination of CAD and DVT; 1, visceral thrombosis). Among these, 1 patient in particular developed CAD during the follow up period.

\section{Thromboelastometry parameters and correlations}

Patients with ET had shorter CFT (median $59 \mathrm{sec}, 33-174$ ), compared to PV (median $82 \mathrm{sec}, 29-156$ ) and MF (median 101.5 sec, 46-272), $\mathrm{p}=0.001$. Furthermore, MCF was higher in ET (median $69 \mathrm{~mm}$ versus $62.5 \mathrm{~mm}$ in PV and $53.5 \mathrm{~mm}$ in $\mathrm{MF}$ ), p=0.0001. In ROTEM", the MCF value is the most commonly used parameter to detect hypercoagulability [22]. Among all patients with high MCF values, only 5/27 (18.5\%) had a history of thrombosis before or after diagnosis. Figure 2 depicts a pathologic ROTEM record from a representative patient with hypercoagulable profile.

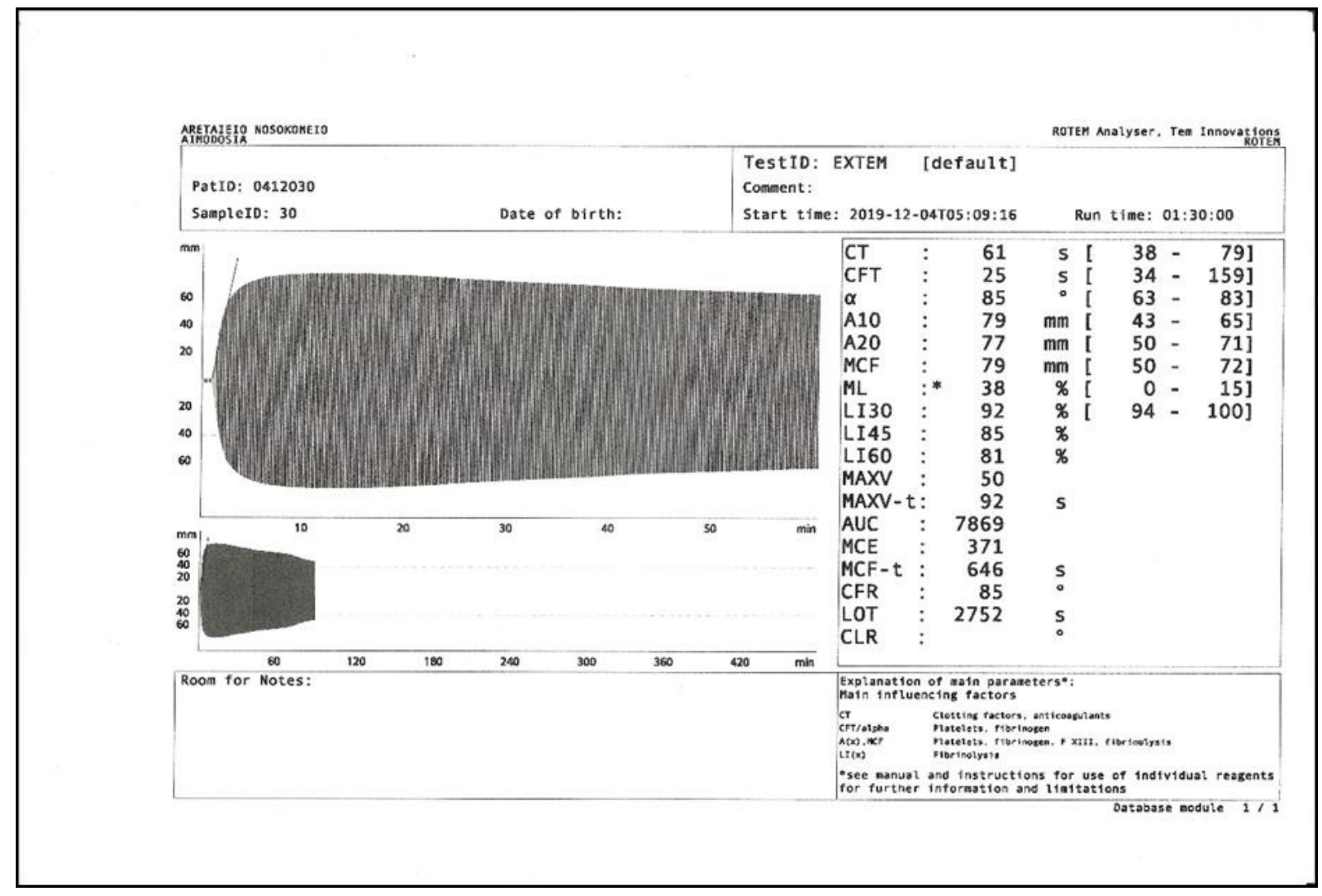

Figure 2: Pathologic ROTEM record of a 70 years old woman with ET under aspirin and a history of DVT before diagnosis 
In all patients, there was a statistically significant positive correlation both between alpha angle and PLT count $\left(r_{s}=0.474, p<0.001\right)$ and between MCF and PLT count $\left(r_{s}=0.680, p<0.001\right)$, (Figures 3 and 4) Moreover, there was a statistically significant strong negative correlation between CFT and PLT count $\left(r_{s}=-0.651, p<0.001\right)$. No correlation was found between CT and PLT count $\left(r_{s}\right.$ $=-0.139, \mathrm{p} \leq 0.154)$.

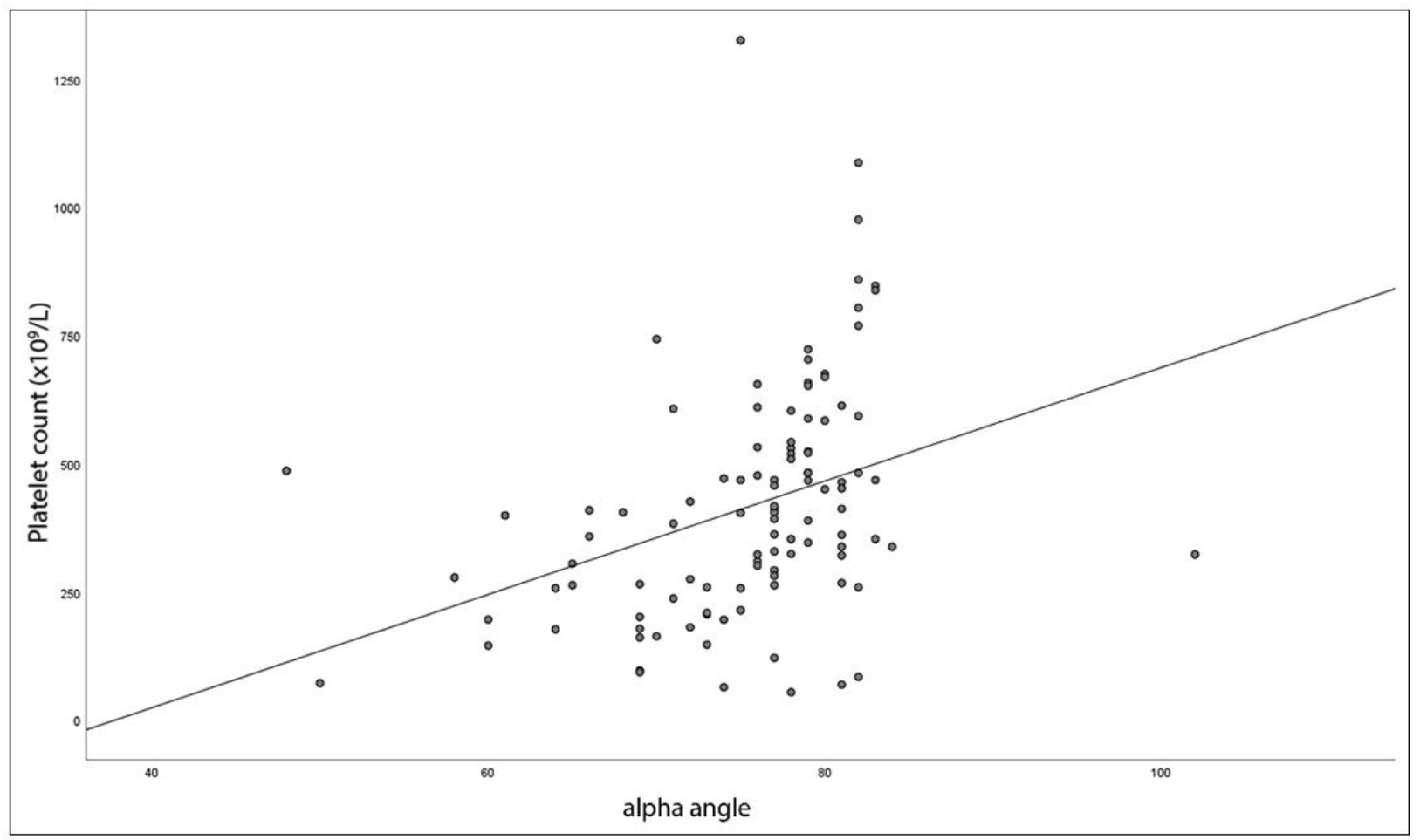

Figure 3: The correlation between platelet count and alpha angle EXTEM $\left(r_{s}=0.474, p<0.001\right)$

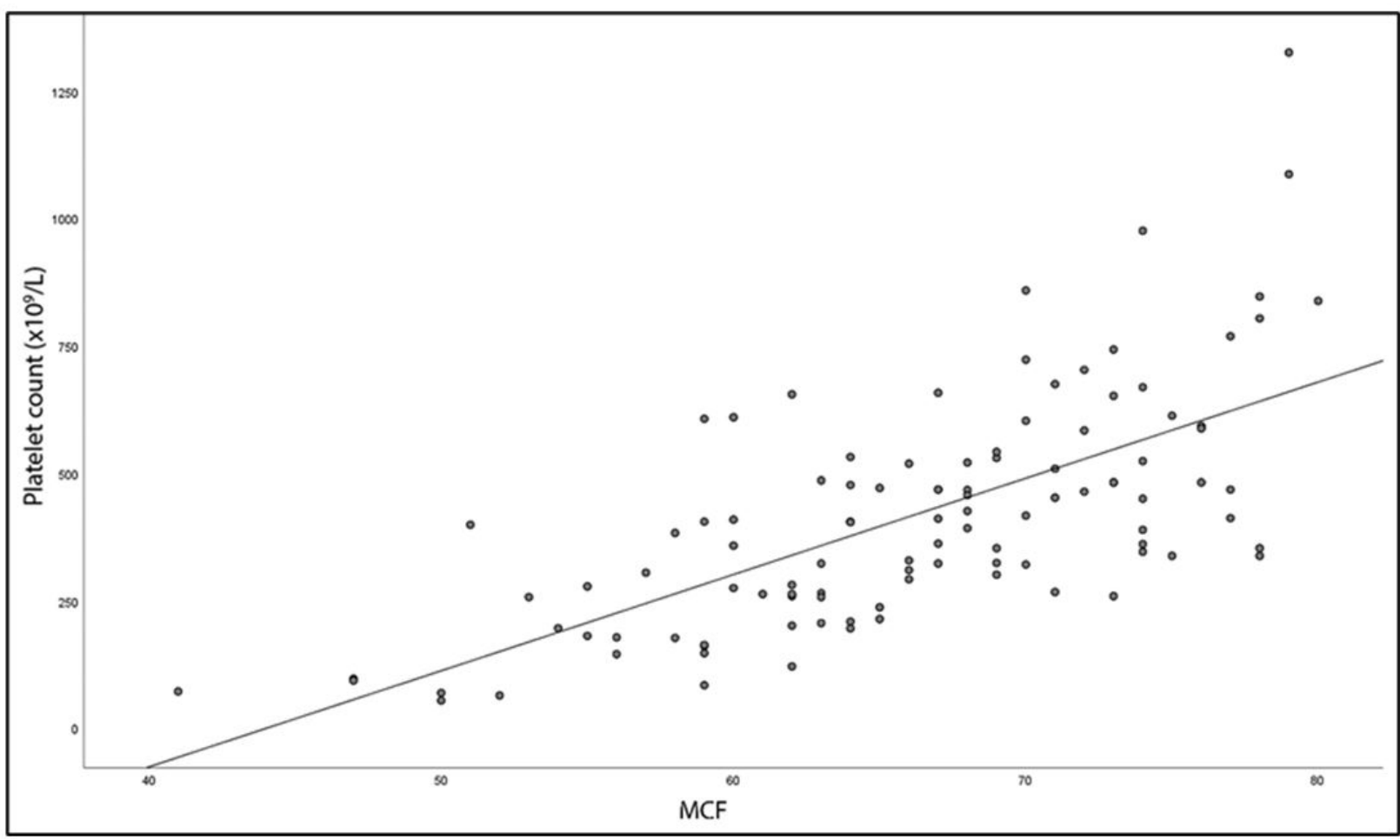

Figure 4: The correlation between platelet count and MCF EXTEM $\left(r_{s}=0.680, p<0.001\right)$ 
The same correlations were confirmed when the subgroup of PV or ET patients were examined separately. Especially in PV patients, a statistically significant positive correlation was found between alpha angle and PLT count $\left(\mathrm{r}_{\mathrm{s}}=0.550, \mathrm{p}=0.002\right)$. Moreover, there was a statistically significant strong negative correlation between CFT and PLT count $\left(r_{s}=-0.699, p<0.001\right)$ and a strong positive one between MCF and PLT count $\left(r_{s}=0.676, p<0.001\right)$. No correlation was found between CT and PLT count $\left(r_{s}=-0.158, p\right.$ $\leq 0.406)$. In ET patients, there was a statistically significant positive correlation between alpha angle and PLT count $\left(r_{s}=0.416, p\right.$ $<0.001)$. A statistically significant strong negative correlation was found between CFT and PLT count $\left(\mathrm{r}_{\mathrm{s}}=-0.602, \mathrm{p}<0.001\right)$ and a strong positive one between MCF and PLT count $\left(r_{s}=0.519, p<0.001\right)$. No correlation was found between CT and PLT count $\left(r_{s}=\right.$ $-0.109, \mathrm{p} \leq 0.391)$. Finally, in MF patients, no correlations were carried out due to the small number of patients.

\section{PARP-1 polymorphisms and correlations}

A statistically significant correlation of the PARP-1 polymorphism rs1136410 with the presence of thrombosis was found in patients with PV. More particularly, none of the patients with this polymorphism had any thrombotic episodes prior to diagnosis (0/10) while none of the patients with thrombotic episodes prior to diagnosis carry the polymorphism (0/7), $\mathrm{p}=0.033$. No patients with thrombosis during follow-up (4/4) had the polymorphism while none of the patients with the polymorphism had a thrombotic episode during follow-up (10/10), $\mathrm{p}=0.083$. Especially in JAK2 positive patients with PV [19/32 (59.3\%)], absence of the PARP-1 polymorphism [11/19 (57.9\%)] was correlated with thrombotic episodes during follow-up [4/19 (21\%)], p=0.019. The presence of the PARP-1 polymorphism in patients with PV was not correlated with age, sex, fibrosis, splenomegaly, fibrinogen, d-dimers, COL/EPI and COL/ADP, thromboelastometry parameters, hemoglobin ( $\mathrm{Hb}$ ), PLT, white blood cell (WBC) counts, mutation status or treatment with any of the recorded agents. Unlike patients with PV, there was no correlation of thrombotic episodes prior or after treatment with the presence of PARP-1 polymorphisms in patients with ET. More specifically, among 17 patients with the polymorphism, 1 patient had thrombosis under treatment while among 48 patients without the polymorphism 7 had a thrombosis under treatment, $(\mathrm{p}=0.666)$. The corresponding values for pretreatment thrombosis are 3/17 and 7/48 respectively, $(\mathrm{p}=0.713)$. In $J A K 2$ positive patients with ET $(\mathrm{N}=32)$, the presence of a polymorphism did not correlate with a thrombosis at baseline ( 1 thrombotic episode among 8 patients with the polymorphism versus 4 episodes among 24 patients without, $\mathrm{p}=1.0$ ) or during follow-up ( 0 thrombosis among 8 patients with polymorphism versus 4 thrombotic episodes among 24 patient without polymorphism, $\mathrm{p}=0.55$ ). Among males with ET, 10/25 were positive for the PARP-1 polymorphism while only 7/40 females were positive for the same polymorphism ( $\mathrm{p}=0.047$, Pearson Chi-Square). The PARP-1 polymorphism in patients with ET was not correlated with age, fibrosis, splenomegaly, d-dimers, COL/EPI and COL/ADP, thromboelastometry parameters, Hb, PLT, WBC or any of the treatments administered.

\section{PFA-100, antiplatelet therapy, and thrombosis}

Correlations were found between the platelet function parameters and the antiplatelet and anticoagulation therapy as well as with the appearance of thrombotic episodes. Among 80 patients under treatment with aspirin at the time of sample collection (73 patients under only aspirin and 7 under a combination with clopidogrel), 47 patients (58.8\%.) had a COL/EPI $\geq 300$ sec while $33(41.2 \%)$ had a COL/EPI <300 sec. Among 9 patients under treatment with clopidogrel (2 patients under only clopidogrel and 7 under a combination with aspirin), only 2 (22.2\%) patients had a COL/ADP $\geq 300$ sec while 7 (77.7\%) had a COL/ADP $<300 \mathrm{sec}$. COL/EPI was $<150 \mathrm{sec}$ in $9 / 80$ (11.25\%) patients receiving aspirin and COL/ADP was $<150 \mathrm{sec}$ in $4 / 9$ (44.4\%) patients receiving clopidogrel. Among those patients with short closure times for COL/EPI and COL/ADP, 1/9 (11.1\%) and 1/4 (25\%) had a thrombotic episode before diagnosis.

Finally, among 47 patients under treatment with aspirin and complete platelet inhibition (COL/EPI $\geq 300 \mathrm{sec}$ ), no patient had a thrombosis during follow-up ( $\mathrm{p}=0.006)$. After diagnosis of PN-MPN, 13 thrombotic episodes were noted in patients. Among these patients, $6 / 13(46.1 \%)$ were under no antiplatelet therapy and $7 / 13$ (53.8\%) were receiving aspirin ( $\mathrm{p}=0.701)$. Among patients under aspirin, 7/7 had COL/EPI <300 sec (median $202.8 \mathrm{sec}, 115-295)$. 


\section{Thrombosis and anticoagulation therapy}

None of these patients with a post-diagnosis thrombosis were under anticoagulation therapy at the time of the thrombotic episode $(p=0.529)$. Nevertheless, none of them had a new thrombotic episode after the initiation of anticoagulants during the follow-up period.

\section{Differences between patients with and without thrombosis}

In 101 (88.5\%) patients not treated with anticoagulants, the PT, INR, aPTT, fibrinogen, and d-dimer levels did not differ between patients with and without a post-diagnosis thrombotic episode (corresponding level of significance as follows; PT, p=0.479; INR, $\mathrm{p}=0.930$; aPTT, $\mathrm{p}=0.187$; Fib, $\mathrm{p}=0.239$; $\mathrm{d}$-dimer, $\mathrm{p}=0.863)$. Similarly, CT $(\mathrm{p}=0.078)$, CFT $(\mathrm{p}=0.946)$, alpha angle ( $\mathrm{p}=0.630), \mathrm{MCF}$ $(\mathrm{p}=0.673)$ did not differ in patients with and without post-diagnosis thrombotic episodes.

LI60 was lower (median $95.0 \%, 64-100$ ) in patients with thrombosis than in patients without thrombosis (median 96.0 \%, 89-100), $\mathrm{p}=0.024$.

\section{Mutational status and thrombosis}

No correlation was found either between mutational status and thrombosis $(\mathrm{p}=0.499)$ or diagnosis $(\mathrm{PV}, \mathrm{ET}, \mathrm{MF})$ and thrombotic episodes $(\mathrm{p}=0.499)$. Thrombosis, in particular, was not correlated with JAK2 mutation $(\mathrm{p}=0.506), C A L R$ mutation ( $\mathrm{p}=0.719)$, or $M P L$ mutation $(\mathrm{p}=0.878)$.

\section{PN-MPN treatment and thrombosis}

Finally, the type of PN-MPN cytoreductive treatment was not correlated with the occurrence of a thrombotic event during followup period [HU $(\mathrm{p}=0.937)$, anagrelide $(\mathrm{p}=0.753)$, ruxolitinib $(\mathrm{p}=0.117)$, interferon alpha $(\mathrm{p}=0.226)$ and alkylating agent $(\mathrm{p}=0.536)]$.

All the parameters that we have examined such as the PARP-1 polymorphism, were tested in correlation with all the clinical and laboratory parameters (age, gender, diagnosis, mutation status, complete blood count, routine coagulation tests, ROTEM', PFA100 , history of thrombotic episodes, cytoreductive therapy, anticoagulation and antiplatelet treatment) as mentioned above in detail. Multifactorial analysis was not conducted because of the absence of well-established prognostic factors of thrombosis and due to the fact that the majority of the patients were under treatment.

\section{Discussion}

The pathogenesis of thrombosis in PN-MPN patients is multifactorial and not well understood yet. Pathophysiological mechanisms probably involve hemodynamic changes, such as increased viscosity, increased levels of microparticles (MPs) with procoagulant activity, and increased cytokine expression leading to a general inflammatory response. Abnormalities of all MPN-clone-derived blood cells also play a role. More specifically, activated PLTs express P-selectin and tissue factor, release MPs, and provide a catalytic surface for the generation of thrombin. Biochemical changes in the cell membrane and content of red blood cells (RBCs) may lead to aggregation of RBCs and impaired blood flow. In addition, activated neutrophils release proteolytic enzymes and reactive oxygen species and can activate or damage PLTs and endothelial cells as well as impair some coagulation proteins [23-25].

Regarding therapy in PN-MPN, low-dose aspirin 75 to $100 \mathrm{mg}$ once daily for thrombosis prevention is a standard of care in lowrisk patients with PV and ET [26,27]. Cytoreductive therapy is reserved for high-risk patients in both ET and PV and low-risk PV patients who suffer from uncontrolled symptoms, symptomatic splenomegaly, and intolerance to phlebotomy [28,29]. 
Anticoagulation therapy, such as vitamin $\mathrm{K}$ antagonists, direct oral anticoagulants, and low-molecular-weight heparin, is recommended for arterial and venous thrombotic episodes and long-term anticoagulation to prevent recurrent thrombosis should be taken into account according to the kind of the episode [30].

A number of studies have provided evidence that the PARP-1 rs1136410 polymorphism may be involved in cancer development, such as gastric, cervical, and lung cancers, among Asians [12,15], but the same polymorphism seemed to reduce the breast cancer risk and delay progression in a Chinese population [16]. Moreover, decreased risk of cancer was found among Caucasians with this polymorphism, particularly of glioma [12,15]. On the other hand, no association has been found between the PARP-1 rs1136410 polymorphism and individual susceptibility for PN-MPN in a Portuguese population [31]. Our study in Caucasians, showed that the presence of this polymorphism in patients with PV was correlated with an absence of thrombosis prior to diagnosis whereas the absence of this polymorphism in JAK2 positive patients with PV was correlated with thrombotic episodes after diagnosis. To our knowledge, this is the first report of such a finding in PV patients. On the other hand, there are few studies correlating PARP-1 to the presence of thrombosis in the general population. The PARP-1 rs2271347 polymorphism was significantly associated with increased ischemic stroke risk in a Chinese population [32] whereas greater PARP activity in circulating leukocytes was found to have a possible causal role in the development of obstructive coronary artery disease among Chinese patients with diabetes mellitus [33].

Global assays, such as thromboelastometry, may be more useful than conventional hemostatic laboratory tests in depicting the hypercoagulable state in oncologic and hematologic diseases [34]. Thorson et al. demonstrated that intra-abdominal malignancies are associated with hypercoagulable ROTEM parameters including decreased CFT, increased alpha angle and increased MCF [35] while Davies et al. noted that, compared to controls, patients with lung cancer have reduced CT, increased MCF and increased alpha angle [36]. In our study, patients with ET were found to have a hypercoagulable profile, characterized by higher MCF and shorter CFT values compared to patients with PV and MF, a finding similar to previous studies. More specifically, Tripodi et al. noted a procoagulant imbalance in ET and MF patients with shorter CFT and higher MCF when compared to controls while the same pattern was not found in PV patients [9]. The hypercoagulable state notably in ET according to ROTEM parameters, was also confirmed in other studied groups with ET patients compared to healthy controls based on CFT and MCF values [10,11]. PN-MPN are characterized by thrombotic complications and ROTEM ${ }^{\circledast}$ could be used in those patients to predict thrombotic risk according to parameters such as MCF [10]. However, in our study only 5 patients with high MCF value experienced a thrombosis in the past. Larger prospective studies would be needed to clarify if ROTEM parameters could predict hemostatic balance and thrombotic risk in PN-MPN patients.

In our study, according to LI60 values, patients with a thrombosis had a slightly bigger hyperfibrinolytic status compared to those without a thrombosis.

Moreover, according to our data, significant correlation was found between certain ROTEM ${ }^{\circ}$ parameters and platelet counts. In particular, patients with PV and ET with high MCF and large alpha angle had high platelets while patients with high CFT values had low platelets. Similar observations have already been published not only in patients with PN-MPN [8,11] but also in patients with solid tumors [22].

PFA-100 may be useful for the monitoring of treatment with antiplatelet agents not only in the general population but also in MPN patients [37,38]. Moreover, in a large single-center study on a Korean general population including patients with malignancies, among patients receiving only clopidogrel a higher frequency of malignancies was found in the group with normal values of COL/ ADP than in the group with prolonged values [39].

In our study, there was evidence that the antiplatelet therapy may not be sufficient in a significant percentage of patients with PN-MPN as $11.25 \%$ of patients under treatment with aspirin had COL/EPI $<150 \mathrm{sec}$ and $44.4 \%$ of patients under treatment with clopidogrel had COL/ADP $<150 \mathrm{sec}$. Furthermore, the PFA-100 assay could be used to identify patients at higher risk for 
thrombosis except from identifying patients who are resistant and don't respond to antiplatelet therapy. Indeed, shorter COL/EPI values as well as shorter COL/ADP values have been linked to higher risk for thrombosis not only in PN-MPN patients but also in patients with acute myocardial infarction [40,41,42]. However, our small sample size could not confirm such finding. Larger studies are needed to fully evaluate these findings and their clinical relevance.

Unlike the findings of other studies where JAK2 mutation especially in ET patients increases the risk of thrombosis [43,44], our data showed that mutational status does not affect the hypercoagulable state of these patients. In addition, this study showed that the type of cytoreductive therapy for PN-MPN patients does not affect their thrombotic profile.

The limitations of the present study are the small number of patients with MF mainly due to the fact that the enrollment of the patients was consecutive and not selective and the fact that hemostatic parameters were assessed only at one time point (the time of the enrollment) and only in MPN patients since a control group was not included.

Based on the results of the present study, we would like to suggest the following for patients with PN-MPN. All PN-MPN patients regardless of their risk group should receive antiplatelet therapy with aspirin and should be considered to have a platelet function assessment with PFA-100. If COL/EPI is especially $<150 \mathrm{sec}$, one could consider the increase of aspirin dose from once to twice daily in order to achieve a better antiplatelet response as showed also by Rocca et al in a randomized multicenter double-blind trial in patients with ET [45,46]. Especially JAK2 positive patients with PV could be assessed for the detection of the PARP-1 polymorphism rs1136410 because the absence of this polymorphism may be a prognostic factor for the development of thrombotic episodes. According to our data that no patient under anticoagulant therapy had a new thrombosis despite the small number of them (only 13/114), we recommend that all high-risk PN-MPN patients under anticoagulants due to a thrombosis should be thought to continue this therapy in addition to cytoreductive therapy throughout the course of their disease due to an increased risk for recurrence of thrombotic episodes especially those with splachnic vein thrombosis [30,47,48].

\section{Conclusion}

The presence of the PARP-1 polymorphism rs1136410 in patients with PV and especially in JAK2 positive ones and its correlation with thrombotic episodes prior to diagnosis or/and after the initiation of therapy should be approached in further larger studies of PN-MPN patients. Global hemostatic assays could be incorporated in predictive scores along with other hematological parameters and mutational status in order to identify patients with PN-MPN at higher risk for thrombosis and to guide clinicians for the type and dose of appropriate treatment (cytoreductive and/or anticoagulants).

\section{Author Contributions}

N.G. analyzed the results, drafted the manuscript, contributed in the conceptualization and methodology; P.D. analyzed the results and contributed in the conceptualization and review of the final manuscript; M.P. contributed in the conceptualization and methodology and reviewed the final manuscript; C.N.K., A.G., D.K., T.K. and K.Z. performed the experiments; S.C., M.D. and M.M. provided clinical samples and reviewed the manuscript; G.K. contributed in the analysis and validation of the results; N.A.V. contributed in the conceptualization and methodology, reviewed and approved the final manuscript. All authors have read and agreed to the published version of the manuscript.

\section{Funding}

This research received no external funding. 


\section{Institutional Review Board Statement}

The study was approved by the Institutional Review Board.

\section{Informed Consent Statement}

Informed consent was obtained from all subjects involved in the study.

\section{Data Availability Statement}

Data is available upon reasonable request.

\section{Acknowledgments}

We would like to thank Ms. Evita C. Alexopoulos for copy-editing the final manuscript.

\section{Conflicts of Interest}

The authors declare no conflict of interest. 


\section{References}

1. Arber DA, Orazi A, Hasserjian R, Thiele J, Borowitz MJ, et al. (2016) The 2016 revision to the World Health Organization classification of myeloid neoplasms and acute leukemia. Blood 127: 2391-405.

2. Helbig G (2018) Classical Philadelphia-negative myeloproliferative neoplasms: focus on mutations and JAK2 inhibitors. Med Oncol 35: 119.

3. Huang X, Wu J, Deng X, Xu X, Zhang X, et al. (2020) Mutation profiles of classic myeloproliferative neoplasms detected by a customized next-generation sequencing-based 50-gene panel. Journal of Bio-X Research 3: 13-20.

4. Hultcrantz M, Björkholm M, Dickman PW, Landgren O, Derolf ÅR, et al. (2018) Risk for Arterial and Venous Thrombosis in Patients With Myeloproliferative Neoplasms: A Population-Based Cohort Study. Ann Intern Med 168: 317-25.

5. Kaifie A, Kirschner M, Wolf D, Maintz C, Hänel M, et al. (2016) Bleeding, thrombosis, and anticoagulation in myeloproliferative neoplasms (MPN): analysis from the German SAL-MPN-registry. J Hematol Oncol 9: 18.

6. Parashar Y, Kushwaha R, Kumar A, Agarwal K, Singh US, et al. (2016) Haemostatic Profile in Patients of Myeloproliferative Neoplasms-A Tertiary Care Centre Experience. J Clin Diagn Res 10: Ec01-ec04.

7. Harahsheh Y, Ho KM (2018) Use of viscoelastic tests to predict clinical thromboembolic events: A systematic review and metaanalysis. Eur J Haematol 100: 113-23.

8. Giaccherini C, Verzeroli C, Marchetti M, Gamba S, Piras F, et al. (2016) PO-26 - Whole blood rotational thromboelastometry (ROTEM) to detect hypercoagulability in patients with myeloproliferative neoplasms (MPN). Thromb Res 140 Suppl 1: S185-6.

9. Tripodi A, Chantarangkul V, Gianniello F, Clerici M, Lemma L, et al. (2013) Global coagulation in myeloproliferative neoplasms. Ann Hematol 92: 1633-9.

10. Şahin DG, Akay OM, Uskudar Teke H, Andıc N, Gunduz E (2021) Use of rotational thromboelastometry for a global screening of coagulation profile in patients of myeloproliferative neoplasms. Platelets 32: 280-3.

11. Giaccherini C, Marchetti M, Verzeroli C, Gamba S, Russo L, et al. (2019) PB2192 global coagulation capacity of essential thrombocythemia patients by rotem analysis adjusted for platelet count correlates with thrombotic risk class. HemaSphere 3: 983.

12. Qin Q, Lu J, Zhu H, Xu L, Cheng H, et al. (2014) PARP-1 Val762Ala polymorphism and risk of cancer: a meta-analysis based on 39 case-control studies. PLoS One 9: e98022.

13. Diamantopoulos P, Zervakis K, Zervakis P, Sofotasiou M, Vassilakopoulos T, et al. (2017) Poly (ADP-ribose) polymerase 1 mRNA levels strongly correlate with the prognosis of myelodysplastic syndromes. Blood Cancer J 7: e533.

14. Diamantopoulos PT, Kontandreopoulou CN, Symeonidis A, Kotsianidis I, Pappa V, et al. (2019) Bone marrow PARP1 mRNA levels predict response to treatment with 5-azacytidine in patients with myelodysplastic syndrome. Ann Hematol 98: $1383-92$.

15. Yu H, Ma H, Yin M, Wei Q (2012) Association between PARP-1 V762A polymorphism and cancer susceptibility: a metaanalysis. Genet Epidemiol 36: 56-65. 
16. Ma X-B, Wang X-J, Wang M, Dai Z-M, Jin T-B, et al. (2016) Impact of the PARP1 rs1136410 and rs3219145 polymorphisms on susceptibility and clinicopathologic features of breast cancer in a Chinese population. Translational Cancer Research 5: 520-8.

17. Wang XB, Cui NH, Zhang S, Guo SR, Liu ZJ, et al. (2017) PARP-1 Variant Rs1136410 Confers Protection against Coronary Artery Disease in a Chinese Han Population: A Two-Stage Case-Control Study Involving 5643 Subjects. Front Physiol 8: 916.

18. Crochemore T, Piza FMT, Rodrigues RDR, Guerra JCC, Ferraz LJR, et al. (2017) A new era of thromboelastometry. Einstein (Sao Paulo) 15: 380-5.

19. Palumbo GA, Stella S, Pennisi MS, Pirosa C, Fermo E, et al. (2019) The Role of New Technologies in Myeloproliferative Neoplasms. Front Oncol 9: 321.

20. Veneri D, Capuzzo E, de Matteis G, Franchini M, Baritono E, et al. (2009) Comparison of JAK2V617F mutation assessment employing different molecular diagnostic techniques. Blood Transfus 7: 204-9.

21. Didone A, Nardinelli L, Marchiani M, Ruiz ARL, de Lima Costa AL, et al. (2016) Comparative study of different methodologies to detect the JAK2 V617F mutation in chronic BCR-ABL1 negative myeloproliferative neoplasms. Pract Lab Med 4: 30-7.

22. Akay OM, Ustuner Z, Canturk Z, Mutlu FS, Gulbas Z (2008) Laboratory investigation of hypercoagulability in cancer patients using rotation thrombelastography. Medical Oncology 26: 358.

23. Landolfi R, Di Gennaro L, Falanga A (2008) Thrombosis in myeloproliferative disorders: pathogenetic facts and speculation. Leukemia 22: 2020-8.

24. Reikvam H, Tiu RV (2012) Venous thromboembolism in patients with essential thrombocythemia and polycythemia vera. Leukemia 26: 563-71.

25. Barbui T, Finazzi G, Falanga A (2013) Myeloproliferative neoplasms and thrombosis. Blood 122: $2176-84$.

26. Patrono C, Rocca B, De Stefano V (2013) Platelet activation and inhibition in polycythemia vera and essential thrombocythemia. Blood 121: 1701-11.

27. Landolfi R, Marchioli R, Kutti J, Gisslinger H, Tognoni G, et al. (2004) Efficacy and safety of low-dose aspirin in polycythemia vera. N Engl J Med 350: 114-24.

28. Fruchtman SM, Mack K, Kaplan ME, Peterson P, Berk PD, et al. (1997) From efficacy to safety: a Polycythemia Vera Study group report on hydroxyurea in patients with polycythemia vera. Semin Hematol 34: 17-23.

29. Cortelazzo S, Finazzi G, Ruggeri M, Vestri O, Galli M, et al. (1995) Hydroxyurea for patients with essential thrombocythemia and a high risk of thrombosis. N Engl J Med 332: 1132-6.

30. Arachchillage DR, Laffan M (2019) Pathogenesis and Management of Thrombotic Disease in Myeloproliferative Neoplasms. Semin Thromb Hemost 45: 604-11.

31. Azevedo AP, Silva SN, De Lima JP, Reichert A, Lima F, et al. (2017) DNA repair genes polymorphisms and genetic susceptibility to Philadelphia-negative myeloproliferative neoplasms in a Portuguese population: The role of base excision repair genes polymorphisms. Oncol Lett 13: 4641-50. 
32. Meng D, He W, Huang P, Liu D, Zhong L, et al. (2018) Polymorphism of PARP-1 indicates an increased risk and a worse initial severity of ischemic stroke. Per Med 15: 355-60.

33. Cui NH, Yang JM, Liu X, Wang XB (2020) Poly(ADP-Ribose) Polymerase Activity and Coronary Artery Disease in Type 2 Diabetes Mellitus: An Observational and Bidirectional Mendelian Randomization Study. Arterioscler Thromb Vasc Biol 40: 251626.

34. Walsh M, Kwaan H, McCauley R, Marsee M, Speybroeck J, et al. (2020) Viscoelastic testing in oncology patients (including for the diagnosis of fibrinolysis): Review of existing evidence, technology comparison, and clinical utility. Transfusion 60 Suppl 6: S86-s100.

35. Thorson CM, Van Haren RM, Ryan ML, Curia E, Sleeman D, et al. (2014) Pre-existing hypercoagulability in patients undergoing potentially curative cancer resection. Surgery 155: 134-44.

36. Davies NA, Harrison NK, Sabra A, Lawrence MJ, Noble S, et al. (2015) Application of ROTEM to assess hypercoagulability in patients with lung cancer. Thromb Res 135: 1075-80.

37. Kitchens CS, Alving BM, Kessler CM (2007) Point-of-Care Testing for Hemostatic Disorders In: Consultative Hemostasis and Thrombosis (2 $2^{\text {nd }}$ Edn) Saunders, Philadelphia, USA.

38. Appelmann I, Kreher S, Parmentier S, Wolf HH, Bisping G, et al. (2016) Diagnosis, prevention, and management of bleeding episodes in Philadelphia-negative myeloproliferative neoplasms: recommendations by the Hemostasis Working Party of the German Society of Hematology and Medical Oncology (DGHO) and the Society of Thrombosis and Hemostasis Research (GTH). Ann Hematol 95: 707-18.

39. Kweon OJ, Lim YK, Kim B, Lee MK, Kim HR (2019) Effectiveness of Platelet Function Analyzer-100 for Laboratory Detection of Anti-Platelet Drug-Induced Platelet Dysfunction. Ann Lab Med 39: 23-30.

40. Tsantes AE, Mantzios G, Giannopoulou V, Tsirigotis P, Bonovas S, et al. (2008) Monitoring aspirin treatment in patients with thrombocytosis: comparison of the platelet function analyzer (PFA)-100 with optical aggregometry. Thromb Res 123: 100-7.

41. Cheung BYY, Parmar K, Yadegarfar G, Hunt BJ, Harrison CN (2005) The Use of the PFA-100 ${ }^{\circledR}$ and Thromboelastograph in Assessing Haemostasis and Drug Efficacy in Essential Thrombocythemia. Blood 106: 4959.

42. Frossard M, Fuchs I, Leitner JM, Hsieh K, Vlcek M, et al. (2004) Platelet function predicts myocardial damage in patients with acute myocardial infarction. Circulation 110: 1392-7.

43. Lussana F, Caberlon S, Pagani C, Kamphuisen PW, Büller HR, et al. (2009) Association of V617F Jak2 mutation with the risk of thrombosis among patients with essential thrombocythaemia or idiopathic myelofibrosis: a systematic review. Thromb Res 124 : 409-17.

44. Cheung B, Radia D, Pantelidis P, Yadegarfar G, Harrison C (2006) The presence of the JAK2 V617F mutation is associated with a higher haemoglobin and increased risk of thrombosis in essential thrombocythaemia. Br J Haematol 132: $244-5$.

45. Rocca B, Tosetto A, Betti S, Soldati D, Petrucci G, et al. (2020) A randomized double-blind trial of 3 aspirin regimens to optimize antiplatelet therapy in essential thrombocythemia. Blood 136: 171-82. 
46. Braunstein EM, Chaturvedi S (2020) Aspirin in ET: will twice a day keep thrombosis away? Blood 136: 151-3.

47. Sekhar M (2017) Prevention and management of thrombosis in myeloproliferative neoplasms. Clin Adv Hematol Oncol 15: 178-81.

48. Hamulyák EN, Daams JG, Leebeek FWG, Biemond BJ, te Boekhorst PAW, et al. (2021) A systematic review of antithrombotic treatment of venous thromboembolism in patients with myeloproliferative neoplasms. Blood Advances 5: 113-21.

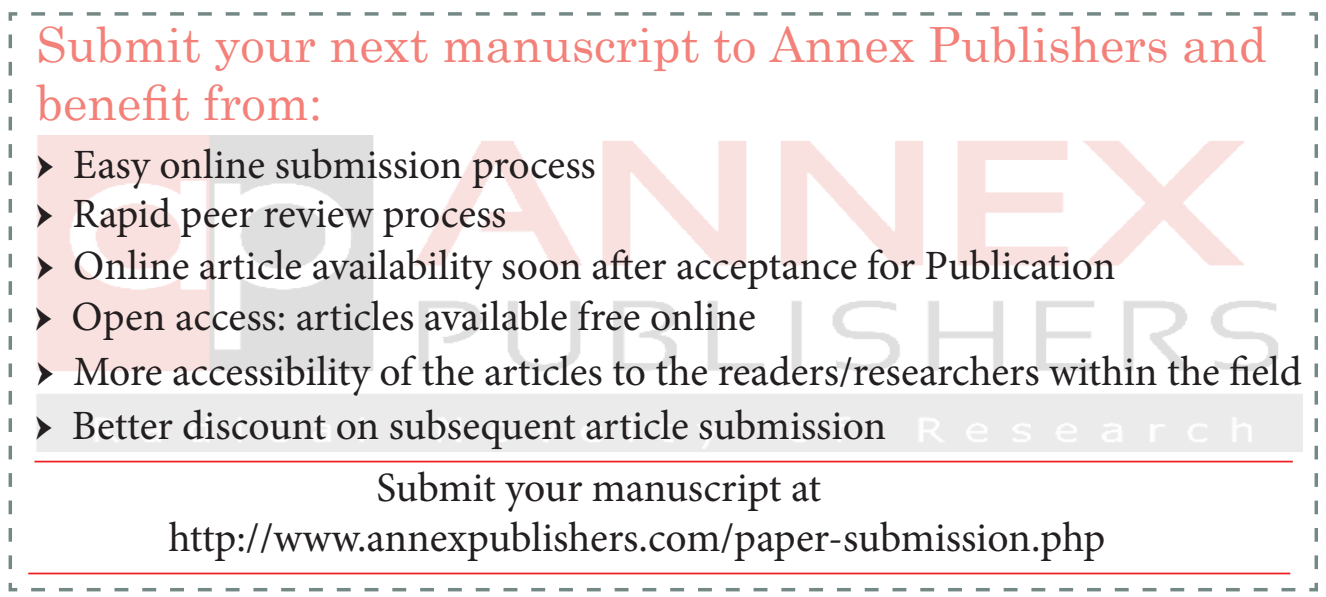

